\title{
Dermatophytes and other fungi associated with skin mycoses in Tripoli, Libya
}

\author{
Dermatophyten und andere Pilze assoziiert mit Dermatomykosen \\ in Tripoli, Libyen
}

M. S. Ellabib ${ }^{1}$, Z. Khalifa ${ }^{2}$ and K. Kavanagh ${ }^{3}$

Key words. Dermatophytes, Candida, Malassezia, dermatophytosis, candidosis, pityriasis, epidemiology, Libya.

Schlüsselwörter. Dermatophyten, Candida, Malassezia, Dermatophytosen, Candidosen, Pityriasis, Epidemiologie, Libyen.

Summary. This study sought to determine the prevalence of skin infections and their causative agents in the Libyan population. Samples were collected from 2224 patients attending the Dermatology Clinics of the Tripoli Medical Centre (TMC) between August 1997 and December 1999 and were submitted to a mycology laboratory for analysis. Diagnosis was confirmed by microscopic examination in 1180 cases $(53.1 \%)$ and the causative agent was isolated and cultured in 1160 cases (52.2\%). Dermatophytes, Malassezia furfur and Candida albicans were the most common etiological agents isolated. Tinea corporis accounted for $45.9 \%$ of cases $(85 \%$ of cases occurred in children below 15 years of age). The frequency of the other clinical types in descending order was pityriasis versicolor 27.8\% (322 cases), candidiosis $13.4 \%$ (156 cases), tinea pedis $8.1 \%$ (94 cases), tinea manuum 2.6\% (30 cases) and tinea barbae $2.2 \%$ (26 cases). Trichophyton violaceum was the most common etiological agent, responsible for $44 \%$ (300 cases) of dermatophyte infections. Malassezia furfur was ranked the second most frequent causative agent being found in $27.8 \%$ of cases, followed by Trichophyton rubrum $13.8 \%$ (160 cases) and Candida albicans 10\% (116 cases). Other species isolated included

${ }^{1}$ Department of Medical Microbiology, Medical College, Al-Fateh University, Tripoli, ${ }^{2}$ Mycology Section, Central Laboratory, Tripoli Medical Centre, Libya, and ${ }^{3}$ Department of Biology, National University of Ireland Maynooth, Co. Kildare, Ireland.

Correspondence: Dr Kevin Kavanagh, Medical Mycology Unit, Department of Biology, NUI Maynooth, Co. Kildare, Ireland.

Tel: (+353)7083859 Fax: (+353)7083845 E-mail: kevin.kavanagh@may.i.e.
Microsporum canis 8.1\% (94 cases), Epidermophyton floccosum $6.6 \% \quad(76$ cases $)$ and Trichophyton mentagrophytes $3.1 \%$ (36 cases).

Zusammenfassung. Diese Studie untersucht die Verbreitung von Hautinfektionen und deren Erreger in der Bevölkerung von Libyen. Proben von 2224 Patienten, die die Dermatologische Klinik des Tripoli Medical Center (TCM) zwischen August 1997 and Dezember 1999 besucht hatten, wurden analysiert. Mikroskopische Untersuchungen bestätigten die Diagnose in 1180 Fällen (53.1\%). Erreger wurden in 1160 Fällen (52.2\%) isoliert. Dermatophyten, Malassezia furfur and Candida albicans waren die am häufigsten isolierten ätiologischen Agentien. Tinea corporis trat auf in $45.9 \%$ der Fälle $(85 \%$ bei Kindern unter 15 Jahren). Andere klinische Typen waren in absteigender Häufigkeit: Pityriasis versicolor 27.8\% (322 Fälle), Candidose 13.4\% (156 Fälle), Tinea pedis $8.1 \%$ (94 Fälle), Tinea manuum 2.6\% (30 Fälle) und Tinea barbae 2.2\% (26 Fälle). Trichophyton violaceum war das am häufigsten auftretende ätiologische Agens bei Dermatophytosen (44\%, 300 Fälle). Den zweiten Rang nahm Malassezia furfur ein, (27.8\% der Fälle), gefolgt von Trichophyton rubrum (13.8\%, 160 Fälle) und Candida albicans (10\%, 116 Fälle). Andere Erreger waren: Microsporum canis $8.1 \%$ (94 Fälle), Epidermophyton floccosum $6.6 \% \quad$ (76 Fälle) und Trichophyton mentagrophytes $3.1 \%$ (36 Fälle).

\section{Introduction}

Superficial mycoses of the skin are amongst the most prevalent human infectious diseases 
observed in clinical practice [1]. The etiological agents comprise the dermatophytes and yeasts responsible for infections including candidosis and pityriasis versicolor [2]. Tinea capitis and tinea corporis are most frequently recorded in children while tinea unguium, tinea pedis and tinea versicolor are more prevalent in adults [3]. Many epidemiological studies have investigated the prevalence of fungi responsible for superficial mycoses in different regions of the world [4-6]. However, with the exception of a few early studies $[7,8]$ the actual prevalence and incidence of fungal diseases and their most common etiological agents among Libyan nationals are unknown. There are no epidemiological data in the literature to ascertain the true incidence of fungal infections and their etiological agents in this population group. An epidemiological study has been performed over a number of years in the Tripoli Medical Centre (TMC) where superficial fungal infections are among the most common skin diseases seen in patients attending the dermatology clinic. The aim of the work presented here was to determine the incidence of the most prevalent fungi associated with skin infections with reference to dermatophytes and yeasts in the district of Tripoli over a period of 28 months.

\section{Materials and methods}

\section{Patient selection}

From August 1997 to December 1999, samples were taken from skin lesions including skin folds, glabrous skin, the hand, feet or face of 2224 patients attending the Dermatology Clinic at the Tripoli Medical Centre. Patient selection was performed following medical examination and the site of sample collection depended upon the nature of the skin lesion. All patients included in this study resided in the city of Tripoli or in the immediate adjoining rural areas. The patient group consisted of 740 males and 420 females (children ranged in age from 1 to 15 years with an average age of 12 years and adults ranged from 16 to 60 years with an average age of 30 years). Male children accounted for 322 cases, female children for 136, adult males for 418 and adult females for 284 cases of culture positive identification.

\section{Sample collection}

All samples consisted of scales and scrapings taken from the rim of lesions using a sterile scalpel blade following cleaning of the affected sites with $70 \%$ (v/v) isopropyl alcohol. Samples were divided into two portions - one for microscopic examination and one for culture. Samples were analysed microscopically and by growth morphology at the Mycology Laboratory of the TMC.

\section{Microscopic Examination}

Microscopic examination of the samples was performed following treatment with an aqueous solution of $20 \%$ potassium hydroxide $(\mathrm{KOH})$. A portion of the sample was placed on a slide and $50 \mu \mathrm{l}$ of $\mathrm{KOH}$ was added. After $5 \mathrm{~min}$, the wet preparation was examined for the presence of fungal elements and their diagnostic morphology.

\section{Fungal Culture}

All samples were cultured on Sabouraud glucose chloramphenicol actidione agar (SCAA), Sabouraud glucose gentamicin chloramphenicol agar (SGCA) and dermatophyte test agar (DTA) (BioMerieux, Marcy-l'Etoile, France) which was made to the manufacturer's instructions. Olive oil $(2 \%)$ was added to SGCA when pityriasis versicolor was suspected. The plates were inoculated with finely divided pieces from the samples and incubated at 25 or $30^{\circ} \mathrm{C}$ for up to 4 weeks. The plates were examined twice-weekly for evidence of growth. Fungal isolates were then subcultured onto Sabouraud and potato glucose agar in Petri dishes. The isolates were examined macroscopically and microscopically following staining with lactophenol cotton blue. The dermatophytes species were identified by gross and microscopic morphology and by in vitro tests, if required, based on the criteria enumerated by Rebell and Taplin [9] and Frey et al. [10].

Candida species were first identified using API 20C AUX Commercial System (BioMerieux, Marcy-l'Etoile, France). All Candida albicans isolates were tested for germ tube production in human serum at $37^{\circ} \mathrm{C}$ as well as for chlamydospores formation on corn meal agar plus Tween 80.

\section{Results}

Samples were obtained from a total of 2224 patients who presented with suspected superficial mycoses during the course of this study. Diagnosis was confirmed by microscopic examination in 1180 cases $(53.1 \%)$, and the causative agents were isolated in 1160 cases $(52.2 \%)$. From the total number of isolates that were identified by culture growth, species of dermatophytes were the most prevalent and accounted for 682 cases 
(58.8\% of all culture positive fungal infections), $M$. furfur and C. albicans were the next most common fungi being isolated in $322(27.8 \%)$ and $116(10 \%)$ cases, respectively. The frequency of skin mycoses among patients attending the dermatology clinic is presented in Table 1. Of all dermatophytes, Trichophyton violaceum was by far the most commonly isolated etiological agent and accounted for 44\% (300 cases) of dermatophyte isolates, while T. rubrum was ranked second as shown in Table 2.

According to the anatomic site involvement of dermatophyte infection, tinea corporis was the predominant clinical form of all ringworm infections observed and it is noteworthy that $85 \%$ of

Table 1. Frequency (percentage) of skin mycoses among patients attending the Dermatology Clinic at TMC

\begin{tabular}{|lc|}
\hline Disease & Number of cases $(\%)$ \\
\hline Tinea corporis & $532(45.9)$ \\
Tinea versicolor & $322(27.8)$ \\
Candidosis & $156(13.5)$ \\
Tinea pedis & $94(8.1)$ \\
Tinea manuum & $30(2.6)$ \\
Tinea barbae & $26(2.2)$ \\
Total isolates & $1160(100)$ \\
\end{tabular}

Table 2. Causative Dermatophytes isolated in 682 patients with ringworm infections (including tinea corporis, tinea pedis, tinea manuum and tinea barbae)

\begin{tabular}{lc|}
\hline Species & Number of isolates (\%) \\
\hline T. violaceum & $300(44)$ \\
T. rubrum & $160(23.5)$ \\
M. canis & $94(13.8)$ \\
E. floccosum & $76(11.1)$ \\
T. mentagrophytes & $36(5.3)$ \\
T. tonsurans & $16(2.3)$ \\
Total & $682(100)$ \\
\hline T.: Trichophyton; M.: Microsporum; E.: Epidermophyton. \\
\hline
\end{tabular}

cases were in children below the 15 years of age. Tinea pedis was observed more frequently in adults and twice as commonly in female patients than males. With the exception of tinea pedis and tinea manuum in which $\mathcal{T}$. rubrum was the predominant isolate, $T$. violaceum was either the most common etiological agent, as in tinea corporis, or ranked second in the other forms of ringworm infection. The frequency of dermatophytic infections according to body site is presented in Table 3. Infection of glabrous skin, such as the chest, neck and back, was most commonly associated with the lipophilic yeast $M$. furfur (pityriasis versicolor). The infection was found to occur more frequently in adult males (250 cases out of 418) than in females (72 cases out of 284). Superficial candidosis was mainly due to $C$. albicans (116 cases) and most patients were females. The most frequently affected sites were the axiallry (66 cases), hand (22 cases), foot (12 cases) and face (12 cases). Other Candida species isolated included Candida parapsilosis (22 cases), Candida glabrata (13 cases) and Candida tropicalis (5 cases).

\section{Discussion}

During the period of this study, samples were collected from 2224 patients attending the TMC and diagnosed as having a superficial fungal infection. Microscopic identification was performed in 1180 cases and the causative agent was identified by culturing in 1160 cases.

Fungi are ubiquitous and no geographical area or any group of people is spared by these organisms. In Libya, superficial mycoses and fungal infections in general are not notifiable by their relatively low incidence and therefore the real prevalence is unknown. However, accurate assessment of the prevalence and etiologic agent is desirable to estimate the size of therapeutic problem and to prevent the transmission and spread of such infections with adequate measures.

Table 3. Anatomic site distribution of dermatophyte species isolated from patients with ringworm infections

\begin{tabular}{|c|c|c|c|c|c|}
\hline Species & Total & $\begin{array}{l}\text { Tinea corporis } \\
\mathrm{n}(\%)\end{array}$ & $\begin{array}{l}\text { Tinea pedis } \\
\mathrm{n}(\%)\end{array}$ & $\begin{array}{l}\text { Tinea manuum } \\
\mathrm{n}(\%)\end{array}$ & $\begin{array}{l}\text { Tinea barbae } \\
\mathrm{n}(\%)\end{array}$ \\
\hline T. violaceum & 300 & $270(50.8)$ & $12(12.8)$ & $8(26.7)$ & $10(38.5)$ \\
\hline T. rubrum & 160 & $78(14.7)$ & $68(72.3)$ & $12(40.0)$ & $2(7.7)$ \\
\hline M. canis & 94 & $70(13.2)$ & $10(10.6)$ & $4(13.3)$ & $10(38.5)$ \\
\hline E. floccosum & 76 & $74(13.9)$ & 0 & $2(6.7)$ & 0 \\
\hline T. mentagrophytes & 36 & $24(4.5)$ & $4(4.3)$ & $4(13.3)$ & $4(15.4)$ \\
\hline T. tonsurans & 16 & $16(3.0)$ & 0 & 0 & 0 \\
\hline Total isolates & 682 & $532(78.0)$ & $94(14.9)$ & $30(4.4)$ & $26(3.8)$ \\
\hline
\end{tabular}

T.: Trichophyton; M.: Microsporum; E.: Epidermophyton. 
The incidence of skin mycoses in our retrospective study was the most predominant of all clinical forms of superficial mycoses including tinea capitis and onychomycosis. Dermatophytes were the most common cause of all culture positive fungal infections involving the skin observed during the period of this study (58.8\%). Tinea corporis was the most prevalent form of ringworm infection and most cases were found in children. The antropophilic fungus T. violaceum was either the primary causative agent in most cases of ringworm infections or ranked second in frequency. The increasing incidence of $T$. violaceum as a major cause of tinea corporis in Libya may be due to the fact that the fungus is indigenous to North Africa and had been found to be the most common cause of tinea capitis among children in Tripoli [11]. T. violaceum is the most common cause of tinea capitis in Tunisia and Egypt [4,12] which are geographically close to Libya. Living conditions, large family size and close contact either directly or by sharing facilities, including combs and towels, is common between family members in Libya and may facilitate transmission. Many children with tinea corporis had been in contact with other infected children, either within their family or at school. The increasing incidence of $T$. violaceum as the major cause of tinea corporis and the second most common cause of tinea pedis may be due to the dispersal of fungus from scalp lesions to the trunk down the feet of the new host as a result of showering or sharing bathing facilities.

The total number of cases of tinea pedis observed in this study may not reflect the true number of patients with such clinical diseases observed at TMC. In many instances, patients were treated after clinical assessment and in the majority of cases of family epidemics only one member of the affected family was seen and had a sample taken for culture.

T. rubrum is the most common cause of tinea pedis world wide $[3,6]$ and has been shown to be so in Libya also. Environmental study has shown that this fungus is the principal fungal pathogen isolated from Tripoli beaches [13]. Tinea barbae and tinea manuum were seen less frequently during this study. T. rubrum and T. violaceum were found to be main causative agent of tinea manuum, while $T$. violaceum and $M$. canis were isolated more frequently from adult males with tinea barbae.

$M$. furfur, the causative agent of pityriasis versicolor, was found to be more common among Libyan adults and was the most commonly encountered superficial mycoses after tinea corporis. The infection occurred more frequently in the months of June to September, when the weather in Libya is hot and humid. Most of the patients affected with this condition were 20-30-year-old-males, most of whom were keen beach visitors engaging in swimming or sports. A similar result was recorded in Saudi Arabia and Tunisia [3, 4]. Cutaneous candidosis was found to be an important condition particularly among females and involved the chest, hand and buttocks. A study on fungal infections of fingernails (Ellabib and Khalifa, unpublished observation) has shown that the prevalence of the yeasts C. albicans and C. parapsilosis was higher in females than dermatophytes. This finding agrees with other studies, which recorded that cutaneous candidosis may be as important as dermatophytes, particular in females [3-5, 14], due to the fact that females usually harbour C. albicans in the vagina or C. parapsilosis on the skin [15].

\section{References}

1 Rudy, S. J. (1999) Superficial fungal infections in children and adolescents. Nurse Pract. Forum. 10 (2), 56-66.

2 Ayler, R. (1994) Ecology and epidemiology of dermatophyte infections. F. Acad. Dermatol. 31, S21-S25.

3 Venugopal, P. V. \& Venugopal, T. V. (1992) Superficial mycoses in Saudi Arabia. Australas 7. Dermatol. 33 (1), 45-48.

4 Ayadi, A., Borgi, N. \& Makni, F. (1993) Prevalence of superficial mycoses in an urban ecosystem in Sfax (Tunisia). Bull. Soc. Pathol Exot 86 (3), 188-189.

5 Korstanje, M. J. \& Staats, C. C. (1995) Fungal infections in the Netherlands: prevailing fungi and pattern of infection. Dermatology 190 (1), 39-42.

6 De Vroey, C. (1985) Epidemiology of ringworm (dermatophytosis). Sem Dermatol. 4, 185-200.

7 Bhakhtaviziam, C., Shafi, M. \& Mehta, M. C. (1984) Tinea capitis in Tripoli. Clin. Exp. Dermatol. 9, 84-88.

8 Malhotra, Y., Garg, M. \& Kanwar, A. (1979) A school survey of tinea capitis in Benghazi, Libya. 7. Trop. Med. Hyg. 82, 59-61.

9 Rebell, G. \& Taplin, D. (1979) Dermatophytes: their recognition and identification. Coral Gables, Florida, USA: University of Miami Press.

10 Frey, D., Oldfield, R. J. \& Bridger, R. C. (1979) A Colour Atlas of Pathogenic Fungi. London: Wolfe Medical Publications Ltd.

11 Ellabib, M., Rafaai, A. \& El-Gamodi, F. (1996) Frequency of occurrence of principal dermatophytes and their causative agents observed in Tripoli hospital. In The Third famahiriya Medical Sciences Conference. pp. 74.

12 Amer, M., Taha, M. \& Tosson, Z. (1981) The frequency of causative dermatophytes in Egypt. Int. F. Dermatol. 20, 431-434.

13 Sabie, F., Ezabie, S. \& Tani, F. (1996) Isolation and identification of pathogenic fungi from beaches sand in Libya. In The Third Famahiriya Medical Sciences Conference. pp. 75.

14 Guiguemde, T. R., Tapsoba, G. P. \& Para, J. L. (1992) Preliminary data on dermatophytosis in Ouagadougou (Burkina Faso). Med. Trop. 52 (2), 151-155.

15 Odds, F. C. (1988) Ecology of Candida and epidemiology of candidosis. In: Odds, F. C. (ed.), Candida and Candidosis, 2nd edn. London: Bailliere Tindall, pp. 77-82. 